\title{
Predictive Lossless Color Image Compression using Arithmetic Operation
}

\author{
Anupam Mukherjee \\ Department of Electronics \& \\ Communication Engineering \\ St. Thomas College of \\ Engineering. \& Technology \\ Kolkata, West Bengal, India
}

\author{
Mitankar Das Sarkar \\ Department of Electronics \& \\ Communication Engineering \\ St. Thomas College of \\ Engineering. \& Technology \\ Kolkata, West Bengal, India
}

\author{
Amiya Halder \\ Department of Computer \\ Science \& Engineering \\ St. Thomas College of \\ Engineering. \& Technology \\ Kolkata, West Bengal, India
}

\begin{abstract}
This paper describes a simple and efficient method of colour image compression/decompression using the fundamental application of subtraction and formation of a dynamic dictionary after three stages of subtraction. Pixels in different segments generated by a chosen mask window are adjusted such that after three stages of subtraction, either all zero or at most one non-zero element is left. The dictionary stores the coordinates and the value of the non-zero elements. Colour transformation that decorralate colour components allow a significant increase in compression ratio achieved by use of standard lossless compression technique applied independently to the each component (RED, GREEN, BLUE). The experimental results show that this algorithm, using the fundamentals of predictive encoding gives a lossless technique of colour image compression. This approach gives better compression ratio than some other lossless techniques and JPEG, JPEG 2000, JPEG LS etc. and the process have $100 \%$ PSNR value.
\end{abstract}

\section{Keywords}

Colour Image, Image Compression, Lossy Compression, Lossless Compression, Dynamic Dictionary.

\section{INTRODUCTION}

The compression of elevation data is fundamental for a number of applications, including storage, transmission, and real time visualization in navigation exercises. The storage and transmission of high resolution elevation information can consume considerable amount of resources and with the increased interest in mapping the earth and having maps for real time navigation, the development of compression techniques to help in theses task is becoming an absolute must.

The purpose of data compression is using less data to express the original data information as far as possible. Image data must be compressed if rapid or real-time transmission or a large amount of storage is required. The research of image data compression is looking for a high compression ratio method and the compressed image must have a suitable signal noise ratio. Image should be restored well after compression and transmission. There are two types of methods in image compression $[1,2,3]$. The first method reduces the amount of data by classifying the same or similar data characteristics and using a few data to describe the raw data information. This compression is generally called lossless compression. The second is to use the visual characteristic of the human eye in a targeted manner to simplify the unimportant data. This compression is generally called lossy compression. People can use this method as long as the lost data has insignificant impact on human receiving effect subjectively. A digital image is obtained by sampling the information at regular intervals (pixel) in either direction to discretize the information. The information for each pixel is stored numerically in the binary base. The number of bits in each pixel represents the precision of the sample. Higher the precision value better would be the picture quality.

Digital images very often have extremely high redundancy due to lesser variation of adjacent pixel values. Similarity can also be noticed in certain components or bit-planes of the image. The efficiency of the image compression algorithm depends on how fast and how best we can exploit this spatial redundancy and spectral redundancy.

Colour images are more complex than gray scale images as instead of a single intensity value for a pixel, each pixel is usually denoted by three component values such as Red, Green and Blue. Compression based methods that are ideal to use for gray scale images can be easily extended to cope with higher dimensionality, although, the increased dimensionality also leads to more computationally expensive process.

Various compression techniques have been developed for lossy $[4,5,6,7,8,9,10]$ and lossless image compression $[11,12,13]$. We proposed an efficient colour image compression algorithm that should yield higher levels of compression as compared to the JPEG standard and other lossless image compression, while ensuring no loss of the quality of image $[7,14,15]$. Main advantages of this proposed method is very simple in implementation and higher compression ratio.

This proposed algorithm is an elegant and simple scheme to identify the spatial redundancy of image data file without sacrificing the quality. This paper presents in fact a possible extension of the previously presented concept of Arithmetic Operation based Predictive Lossless [16] of gray level images. The basic concept for designing this scheme came from the discipline of subtraction and dynamic dictionary, using the basics of predictive encoding. The lossless predictive encoding/decoding mechanism is shown in Figure 1. 


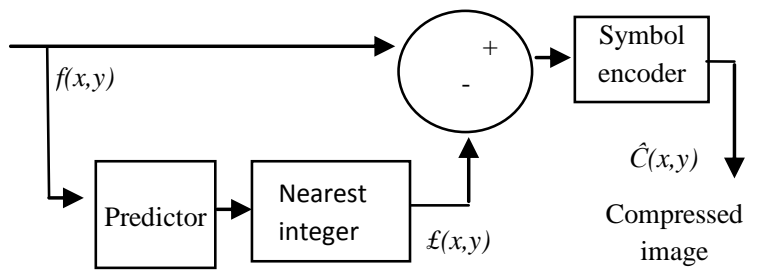

Figure 1(a): Predictive encoding

The rest of this paper is organized as follows:- Section 2, provides the concepts of proposed method. Section 3 describes the experimental results and section 4 concludes the paper.

\section{BASIC CONCEPT OF PROPOSED METHOD}

In an image there is usually a likelihood of high correlation between neighboring pixels. Such correlations between pixels or a block of pixels are exploited to achieve image compression. The basic approach we have formulated for compressing image has been derived from the concept of three stage subtraction, incorporating the basics of predictive encoding.

The function of a basic predictor is to generate the expected value of a given sample (or data) from a previously found out sample database. The output of the predictor is then rounded off to get the nearest integer. As this transformation is invertible and deterministic, it is possible to get back the original image from the error signal. The main purpose of the transformation is to modify the two dimensional image into a form that is easily possible to encode. It is easier to perform encoding on the error pattern than the original image.

In this proposed method, an image to be compressed is divided into non-overlapping blocks of size $2 \times 2$. Compression is achieved by representing each block by four different intensities values corresponding to block size $2 \times 2$ which is obtained by a partitioning based on block statistics.

For every block $\mathrm{B}$, let pixels are $\mathrm{x} 1, \mathrm{x} 2, \mathrm{x} 3, \mathrm{x} 4$ and the corresponding pixel intensities $\left\{\mathrm{f}_{\mathrm{R}}(\mathrm{x} 1), \mathrm{f}_{\mathrm{G}}(\mathrm{x} 1), \mathrm{f}_{\mathrm{B}}(\mathrm{x} 1)\right\}$, $\left\{f_{R}(x 2), f_{G}(x 2), f_{B}(x 2)\right\},\left\{f_{R}(x 3), f_{G}(x 3), f_{B}(x 3)\right\}$ and $\left\{f_{R}(x 4)\right.$, $\left.\mathrm{f}_{\mathrm{G}}(\mathrm{x} 4), \mathrm{f}_{\mathrm{B}}(\mathrm{x} 4)\right\}$. Select the minimum intensity value of block $\mathrm{B}$ for each components $R, G, B$, say $\left.\left\{f_{R}(x 3), f_{G}(x 3), f_{B}(x 3)\right\}\right)$. Subtract the intensity value $\left\{\mathrm{f}_{\mathrm{R}}(\mathrm{x} 3), \mathrm{f}_{\mathrm{G}}(\mathrm{x} 3), \mathrm{f}_{\mathrm{B}}(\mathrm{x} 3)\right\}$ of the pixel $\mathrm{x} 3$ from the corresponding four pixel intensity values. Now if every block has same intensity, the block will be zero. If every block has two different intensity values, the block may have one value. The objective of this image compression technique that each block will have only one non-zero value or all are zero. It is desired that if four pixel are of different intensity value, then after consecutive maximum three subtraction the block will be one non-zero value or all are zero, say, non zero intensity value $\left\{\mathrm{f}_{\mathrm{R}}(\mathrm{x}), \mathrm{f}_{\mathrm{G}}(\mathrm{x}), \mathrm{f}_{\mathrm{B}}(\mathrm{x})\right\}$ and coordinate value is $\mathrm{x} 1, \mathrm{y} 1$. Every non-zero value is stored in dynamic dictionary (DD). Every dynamic dictionary has two
Compressed image

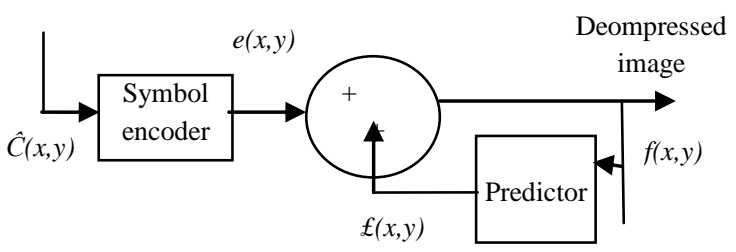

Figure 1(b): Predictive decoding entries, one position and second is value. For all block B, either one non-zero value (from DD) or zero value is stored in two dimensional array of size $(\mathrm{M} \times \mathrm{N}) / 4$. The DD is acting as the predictor in this algorithm. This 2-Dimensional array is the resultant compressed image. The next section describes the details of the proposed algorithm.

\subsection{Image Encoding}

The aim of the scheme is to exploit the correlation between neighboring pixels. The entire image is divided into three planes-Red, Green, Blue. Each plane is further divided into blocks having four pixels each. In the encoding phase of this image compression technique, each plane of size $\mathrm{M} \times \mathrm{N}$ is partitioned into non-overlapping blocks $F=\left\{f_{1}, f_{2}, f_{3}, . . f_{p}\right\}$ of size $2 \times 2$. Every $2 \times 2$ block is represented by $f_{k c}(i, j)$, where $\mathrm{i}=0$ to $1, \mathrm{j}=0$ to $1, \mathrm{c}$ ranges over Red, Green, Blue and $k=1$ to $p$. Calculate the non-zero minimum intensity value $\mathrm{M}_{\mathrm{kc}}$ of the all pixel values in every block

$$
M_{k c}=\min \left(f_{k c}(i, j)\right)
$$

where $\mathrm{c}$ is represents the red, green and blue components.

Now, every block replaced by its modified pixel values. It is modified by the subtraction of the minimum value $\mathrm{M}_{\mathrm{kc}}$ from the original pixel values which is defined as

$$
f_{k c}^{\prime}(i, j)=\left(f_{k c}(i, j)-M_{k c}\right)
$$

After this step, the image is replaced by its new modified value of each block. The reconstructed image is $F_{c}^{\prime}=\left\{f_{1 c}^{\prime}, f_{2 c}^{\prime}, f_{3 c}^{\prime} \ldots . f_{p c}^{\prime}\right\}$ and store the minimum pixel value of every block $\mathrm{M}_{\mathrm{kc}}$ in the $2 \times 2$ window $w_{k c}(i, j)$, where $\mathrm{i}=0$ to $1, \mathrm{j}=0$ to 1 , where $\mathrm{c}$ ranges over red, green, blue and $\mathrm{k}=1$ to $\mathrm{p}$. Now, the new matrix is constructed same as the original size $W_{c}=\left\{w_{1 c}, w_{2 c}, w_{3 c}, \ldots w_{p c}\right\}$ for decompression. 
The next step is applying the similar technique of step 1 on image $F_{c}^{\prime}$, the reconstructed image is $F^{\prime \prime}{ }_{c}=\left\{f_{1 c}^{\prime \prime}, f_{2 c}^{\prime \prime}, f_{3 c}^{\prime \prime} \ldots f_{p c}^{\prime \prime}\right\}$ and minimum intensity values $\mathrm{M}_{\mathrm{kc}}$ of every block of $F^{\prime}{ }_{c}$ is added with the intensity values of the matrix $W_{c}=\left\{w_{1 c}, w_{2 c}, w_{3 c}, \ldots w_{p c}\right\}$ corresponding to the position of the non zero intensity values in the matrix, $F^{\prime}{ }_{c}$.

In the last step is similar to the previous two step, the image $F^{\prime \prime}{ }_{c}$ is modified by the new modified pixel values. For every block of image $\left(f_{1 c}^{\prime \prime \prime}, f_{2 c}^{\prime \prime \prime}, f_{3 c}^{\prime \prime \prime} \ldots f_{p c}^{\prime \prime \prime}\right)$ has one non-zero value or all are zero value, where $\mathrm{c}$ ranges over red, green, blue. The only non-zero value for every block and its position are stored in dynamic dictionary (DD). The total number of nonzero intensity value and its corresponding position are stored in Dynamic Dictionary. After the last step every block of $f_{k c}^{\prime \prime \prime}$ has only one non-zero value or zero value, every nonzero value or one zero value per block is constructed the final compressed image $F^{\prime \prime \prime}{ }_{c}$ and minimum intensity values $\mathrm{M}_{\mathrm{kc}}$ of every block of $F^{\prime \prime}{ }_{c}$ is added with the intensity values of the matrix $W_{c}=\left\{w_{1 c}, w_{2 c}, w_{3 c}, \ldots w_{p c}\right\}$ corresponding to the position of the non zero intensity values in the matrix, $F^{\prime \prime}$.

\subsection{Image Decoding}

For the decoding algorithm of the proposed methods pixel values from the DD are added with the pixel values of $W_{c}($ formed during compression). This is done with accordance with the positioned co-ordinates saved in DD, corresponding to the pixel values. $\mathrm{D}(\mathrm{i}, \mathrm{j})$ is found in this way, where $\mathrm{i}=0$ to (M-1) and $\mathrm{j}=0$ to (N-1) and $\mathrm{c}$ ranges over red, green, blue. The three color planes are then fused together to get an image. This image is same as the original image.

\section{EXPERMENTAL RESULT}

In this section, the result of proposed method is presented and compared with some other well-known techniques. These methods are applied on a number of 24-bit colour images of different sizes. Three of these images are shown in Figure 2. The algorithm developed has been simulated using Visual $\mathrm{C}++$ and MATLAB. Figure 3 gives the lists of coding rates of the proposed scheme together with those of the lossless coding scheme: Row by Row and LZW Encoding [12], JPEGLS and JPEG 2000. It is demonstrated that the proposed scheme attains the best coding performance for all tested images and its coding rates are better than those of the above mentioned techniques.

Here compression ratio measured in terms of bpp and the image quality in terms of PSNR. The bpp and PSNR may be defined, respectively, as

$$
\operatorname{bpp}(\text { bit / pixel })=\frac{24 \times \text { compressed file size }}{\text { Actual file size }}
$$

and

$$
P S N R=20 \times \log \frac{255}{R M S E}, \text { where }
$$

$$
R M S E=\sqrt{\frac{1}{M N} \sum_{i=1}^{M} \sum_{j=1}^{N}\left\|f_{k}(i, j)-\widehat{f}_{k}(i, j)\right\|^{2}}
$$

Where $M$ : number of row, $N$ : number of column, $f(i, j)$ : original image matrix, $\hat{f}(i, j)$ : output image matrix, and $\mathrm{k}$ ranges over Red, Green, Blue. Figure 2 shows the resultant decompressed images.

\section{CONCLUSION}

A new colour image compression algorithm for still images that can be used to compress different type of images has been introduced. The proposed compression scheme is comparable to the jpeg standard and other lossless techniques, compression ratio and corresponding quality. The compression ratio is better than a jpeg standard and other algorithm. However this is one of our future concerns where we would like to include such functionalities.

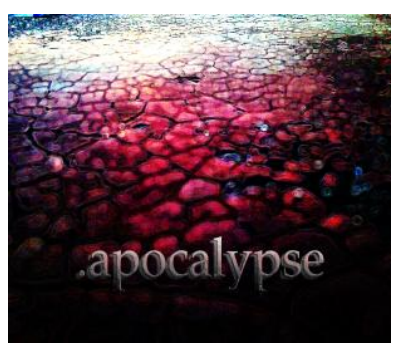

(a)

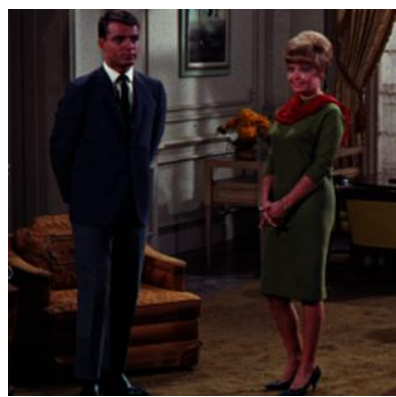

(c)

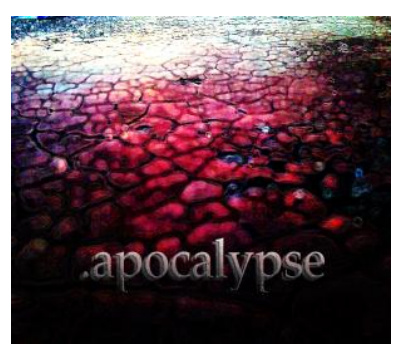

(b)

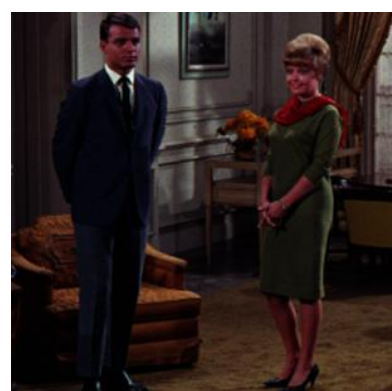

(d) 


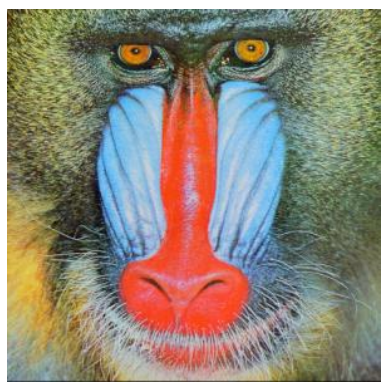

(e)

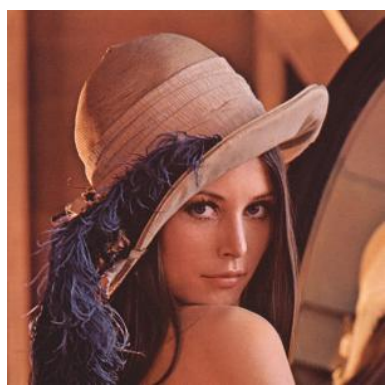

(g)

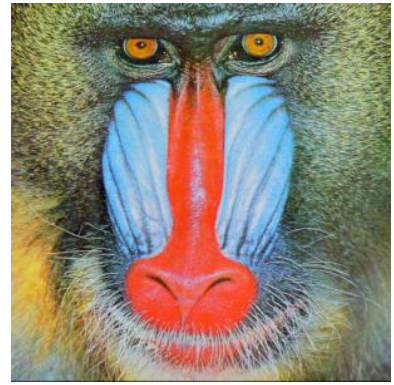

(f)

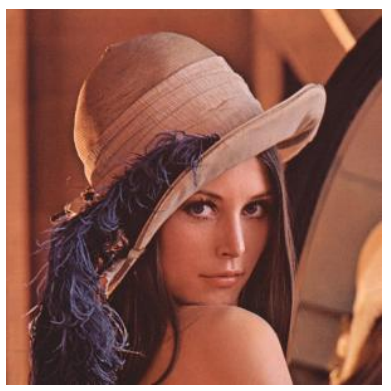

(h)
Figure 2: Some Natural original image (Apocalypse (a), Couple (c), Satellite (e) and Lena (g) ) and decompressed image ((b),(d), (f) and (h)) by proposed method

\section{REFERENCES}

[1] Pratt, William K. " Digital Image Processing”.

[2] Rafael C. Gonzalez, Richard E. Woods, Digital Image Processing, Pearson Education, 2002.

[3] David Saloman, "Data Compression".

[4] S. Bhattacharjee, S,. Das, D. Roy Choudhury and P. Pal Chouduri," A Pipelined Architecture Algorithm for Image Compression”, Proc. Data Compression Conference, Saltlake City, USA, March 1997.

[5] Jorg Ritter and Paul Molitor, "A pipelined architecture for partitioned DWT based lossy image compression using FPGA's," International Symposium on FPGA, pages 201-206,2001.
[6] Amiya Halder, Dipak Kumar Kole and Subarna Bhattacharjee," Online Colour Image Compression based on Pipelined Architecture'ICCEE-2009, Dubai, UAE, Dec 28 - 30,2009.

[7] Tinku Acharya, Ping-Sing Tsai. JPEG2000 Standard for Image Compression.

[8] E. J. Delp and O. R. Mitchell, "Image compression using block truncation coding," IEEE Transactions on Communications, 27:1335-1342, 1979.

[9] J. Jiang, Image compression with neural networks A survey, Image Communication, ELSEVIER, Vol. 14, No. 9, 1999.

[10] Amiya Halder, Sourav Dey, Soumyodeep Mukherjee and Ayan Banerjee, "An Efficient Image Compression Algorithm Based on Block Optimization and Byte Compression", ICISA-2010, Chennai, Tamilnadu, India, pp.14-18, Feb 6, 2010.

[11] Sami Khuri and Hsiu-Chin Hsu "Interactive Packages for Learning Image Compression Algorithms" lists, requires prior specific permission and/or a fee. ITiCSE 2000, Helsinki, Finland.

[12] XIE Yao-hua, TANG Xiao-an, SUN Mao-yin, "Image Compression Based on Classification Row by Row and LZW Encoding”, 2008 Congress on Image and Signal Processing, pp-617-621, 2008.

[13] Debasis Chakraborty and Amiya Halder, " An Efficient Lossless Image Compression Using Special Character Replacement", ICCET 2010, Jodhpur, Rajasthan.

[14] G. K. Wallace, "The JPEG still picture compression standard," Commun. ACM, vol. 34, pp. 31-44, April 1991.

[15] W. B. Pennebaker and J. L. Mitchell, “JPEG: Still Image Data Compression Standard", Van Nostrand Reinhold, New York, 1993.

[16] Mitankar Das Sarkar, Anupam Mukherjee and Amiya Halder, "Arithmetic Operation based Predective Lossless Image Compression", ICSCCN 2011, IEEE Xplore, Tamilnadu, India.

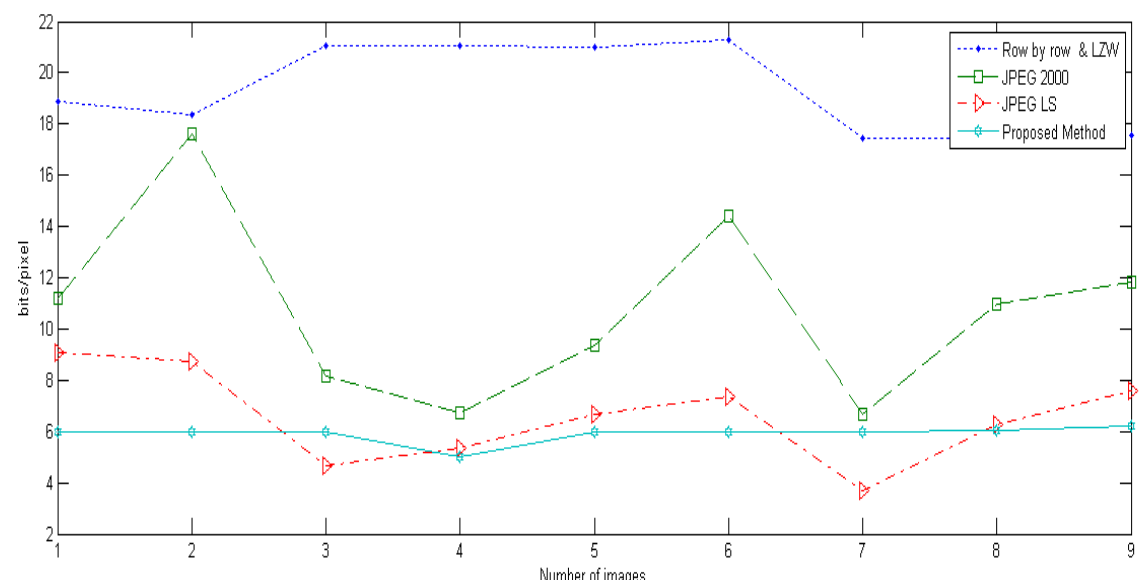

Figure 3: Bit/pixel of the nine natural images by Row by row \& LZW, JPEG 2000, JPEG LS AND proposed method 